Mathematical Research Letters 1, 159-166 (1994)

\title{
THE FIRST EIGENVALUE OF ANALYTIC LEVEL SURFACES ON SPHERES
}

\author{
Sagun Chanillo
}

\begin{abstract}
A BStRACT. In this paper we establish a lower bound for the first eigenvalue of the Laplace-Beltrami operator of the level set of a real valued real-analytic function defined on spheres. The question of existence of such a lower bound was posed by P.Cordaro and J.Hounie and arose in their work on local solvability of systems of vector fields $[\mathrm{CH}]$.
\end{abstract}

\section{Introduction}

The aim of this note is to give a proof of a question raised by Paulo Cordaro and Jorge Hounie. Consider a real valued, real-analytic funtion $f$ defined on the $n+1$ dimensional sphere $S^{n+1}$, with $n \geq 1$, i.e. $f: S^{n+1} \rightarrow \mathbb{R}, \quad n \geq 1$. We next consider the non-singular connected level set $V_{t}$ given by $V_{t}=f^{-1}(t), t \in \mathbb{R}$. $V_{t}$ is a manifold and furthermore $V_{t}$ inherits the Riemannian structure of the sphere. Let $t_{0}$ denote a critical value of $f$. We are interested in the first eigenvalue $\lambda_{1}\left(V_{t}\right)$ as $t \rightarrow t_{0}$, of the Laplace-Beltrami operator on $V_{t}$. We henceforth choose the sign of the Laplace-Beltrami operator on $V_{t}$ so that its eigenvalues are non-negative.

Theorem. There exist constants (independent of $t$ and $t_{0}$ ), $C=C(f)>0$ and $\alpha=\alpha(f)$, such that

$$
\lambda_{1}\left(V_{t}\right) \geq C\left|t-t_{0}\right|^{\alpha} \quad \text { as } t \rightarrow t_{0} .
$$

In the case where $f$ is a polynomial, M. Gromov $[\mathrm{G}]$ has answered the Cordaro-Hounie question in the affirmative. However from the viewpoint of applications to local solvability which originally led Cordaro and Hounie to pose this question, it is essential that $f$ be allowed to be at least realanalytic. When one wishes to study local solvability of a system of vector fields as in $[\mathrm{CH}]$ one is interested in appropriate bounds on the Green

Received September 2, 1993.

Supported by NSF grant DMS-9202051. 
function, this is equivalent to our theorem. The function $f$ arises from the first integrals of these vectors fields which a-priori need not be polynomials. The results of this paper can be used to give another proof of theorem (3.1) of $[\mathrm{CH}]$ wherein Cordaro and Hounie partially proved a conjecture of Treves $[\mathrm{Tr}]$ for the particular case of forms of top degree.

It is possible to prove the theorem by using elementary calculus but this approach leads to a very large value of $\alpha$. For local solvability questions the value of $\alpha$ is immaterial. Since the value of $\alpha$ may be important for other problems we have approached this problem by using some differential geometry, though elementary.

In what follows $C, C_{1}$ denote constants which are different at different places but at all times independent of $t$ and $t_{0}$. In particular $C_{1}$ will only depend on the dimension.

\section{Proof of the theorem}

We proceed by reducing the problem to essentially a Euclidean case. First notice, from the Lojasiewicz inequality,

$$
\inf _{x \in V_{t}}|\nabla f(x)| \geq C\left|t-t_{0}\right|^{\alpha_{1}}
$$

for $C=C(f)>0$ and $\alpha_{1}=\alpha_{1}(f)$ independent of $t$ and $t_{0}$.

We now set up some notation. We will denote by $\left\{e_{1}, \ldots, e_{n}\right\}$ an orthonormal basis for the tangent space at a point $x \in V_{t}$. We denote the sectional curvature at $x$ with respect to the tangent directions $e_{i}$ and $e_{j}$ by $\kappa(x)\left(e_{i}, e_{j}\right)$. Finally we set $|\kappa(x)|=\sup _{i, j}\left|\kappa(x)\left(e_{i}, e_{j}\right)\right|$.

Lemma 1. Let $\kappa(x)$ be defined as above. Then for some $\beta<0, \beta$ independent of $t$ and $t_{0}$, we have

$$
\sup _{x \in V_{t}}|\kappa(x)| \leq C\left|t-t_{0}\right|^{\beta}, \quad C=C(f) .
$$

Proof. This follows from the definition of sectional curvature and (1). In fact one can set $\beta=-3 \alpha_{1}$.

Lemma 2. Let $\tau=\tau\left(V_{t}\right)$ denote the injectivity radius of $V_{t}$. Then

$$
\tau\left(V_{t}\right) \geq C\left|t-t_{0}\right|^{\gamma}, \quad C=C(f) .
$$

Proof. We first note that on $V_{t}$ we have, in suitable local coordinates, $f\left(x_{1}, \ldots, x_{n+1}\right)=t$. Denote by $z_{0}$ any point of $V_{t}$ that we keep fixed for the rest of the calculation. Assume, with no loss of generality in view of 
(1), that $\left|\frac{\partial f}{\partial x_{n+1}}\left(z_{0}\right)\right| \geq C\left|t-t_{0}\right|^{\alpha_{1}}$ and $x_{n+1}$ is the normal direction to the surface $V_{t}$ at $z_{0}$. We will show that there is a neighbourhood of $z_{0}$ of radius at least $C\left|t-t_{0}\right|^{\alpha_{1}}$ such that for all points $z \in V_{t}$ in this neighborhood we also have $\left|\frac{\partial f}{\partial x_{n+1}}(z)\right| \geq C\left|t-t_{0}\right|^{\alpha_{1}}$. Thus the implicit function theorem will tell us that $V_{t}$ is given by a graph in a neighborhood of $z_{0}$ of radius $C\left|t-t_{0}\right|^{\alpha_{1}}$.

To see this, note that

$$
\left|\frac{\partial f}{\partial x_{n+1}}\left(z_{0}\right)\right| \leq\left|\frac{\partial f}{\partial x_{n+1}}\left(z_{0}\right)-\frac{\partial f}{\partial x_{n+1}}(z)\right|+\left|\frac{\partial f}{\partial x_{n+1}}(z)\right| .
$$

Now by (1) applied to the left side of the above inequality and the fact that $f$ is real analytic, in particular $C^{2}$, we have

$$
C\left|t-t_{0}\right|^{\alpha_{1}} \leq C\left|z_{0}-z\right|+\left|\frac{\partial f}{\partial x_{n+1}}(z)\right|
$$

Thus for $\left|z-z_{0}\right| \leq \frac{C}{2}\left|t-t_{0}\right|^{\alpha_{1}}$ our claim follows by subtracting the first term on the right above from the left hand side of the inequality. Next note that the fact that $\left|\frac{\partial f}{\partial x_{n+1}}(z)\right| \geq C\left|t-t_{0}\right|^{\alpha_{1}}$ for all $z$ for which $\left|z-z_{0}\right| \leq\left|t-t_{0}\right|^{\alpha_{1}}$ easily implies that the absolute value of the dot product of the unit normal vector to the graph at $z$ and the unit vector along the positive $x_{n+1}$ axis is bounded below by $C\left|t-t_{0}\right|^{\alpha_{1}}$ for all $z$ for which $\left|z-z_{0}\right| \leq C\left|t-t_{0}\right|^{\alpha_{1}}$. Thus by a projection onto the tangent plane of $V_{t}$ at $z_{0}$, the lemma follows in fact with the choice $\gamma=2 \alpha_{1}$. We point out that the lemma is stated in $[\mathrm{G}]$ with no proof.

We now select $\alpha_{2}$ so that we have

$$
r_{0}=\frac{C\left|t-t_{0}\right|^{\alpha_{2}}}{100} \leq \min \left(\frac{\tau\left(V_{t}\right)}{100}, C\left|t-t_{0}\right|^{-\beta / 2}\right) .
$$

A choice $\alpha_{2}=2 \alpha_{1}$ will suffice, for example. Consider the geodesic ball $B(x, s), s \leq 10 r_{0}$. By Lemma 2 and the choice for $r_{0}$, on this ball we may introduce geodesic polar coordinates. That is, for each $z \in B(x, s)$, we have $z=e^{r \xi}, \quad r \in \mathbb{R}, \quad 0 \leq r \leq 10 r_{0}, \quad \xi \in S^{n-1}$. Let $d \mu$ denote the volume element on $V_{t}$, with respect to the induced metric. Then one has, in geodesic polar coordinates,

$$
d \mu(r, \xi)=\sqrt{g(r, \xi)} d r d \sigma(\xi)
$$

where $d \sigma(\xi)$ is the surface measure of the $n-1$ dimensional sphere. 
Lemma 3. For $r \leq 10 r_{0}$, there exists $C_{1}>0$ a dimensional constant such that

$$
C_{1} r^{n-1} \leq \sqrt{g(r, \xi)} \leq C_{1}^{-1} r^{n-1}
$$

Proof. The left hand inequality follows by using Lemma 1 and the Bishop comparison theorem, eqn. (34), pg. 69 of [C]. We now prove the right hand inequality. First we denote the Ricci tensor by $\operatorname{Ric}(x)\left(\eta, \eta^{\prime}\right)$ where $\eta$ and $\eta^{\prime}$ are two tangent vectors in the tangent space at $x \in V_{t}$. Set $\eta=|\eta| e_{n}$. Here $|\eta|$ denotes the metric length of the tangent vector $\eta$. Then observe that,

$$
\operatorname{Ric}(x)(\eta, \eta)=|\eta|^{2} \sum_{i=1}^{n-1} \kappa(x)\left(e_{i}, e_{n}\right) .
$$

Applying the lower bound of Lemma 1 we see at once that uniformly for $x \in V_{t}$ we have the following lower bound on the Ricci curvature,

$$
\operatorname{Ric}(x)(\eta, \eta) \geq-C(n-1)\left|t-t_{0}\right|^{\beta}|\eta|^{2} .
$$

Thus the second Bishop comparison theorem equation (42), pg. 72 of $[\mathrm{C}]$ applies and we immediately conclude the right hand inequality above. Notice the right hand inequality holds under a weaker hypothesis: just a lower bound for the Ricci curvature will suffice. Lemma 1 gives us a much stronger hypothesis than what is needed to apply the Bishop comparison theorem.

Our next lemma is a local Poincaré inequality. We set up some notation. Let

$$
|B(x, s)|=\frac{s^{n}}{n} \int_{S^{n-1}} d \sigma(\xi),
$$

the "Euclidean" volume of $B(x, s)$. Further, for $z \in B(x, s), z=e^{r \xi}$ we will write $h(z)$ as $h(r, \xi)$.

Lemma 4. For any function $h \in C^{1}\left(V_{t}\right)$, we have, for $0 \leq s \leq 10 r_{0}$ and $C_{1}=C_{1}(n)$,

$$
\int_{B(x, s)}\left|h-h_{B}\right|^{2} d \mu \leq C_{1} s^{2} \int_{B(x, s)}|\nabla h|^{2} d \mu
$$

where $h_{B}=\frac{1}{|B(x, s)|} \int_{B(x, s)} h(r, \xi) r^{n-1} d r d \sigma(\xi)$.

Proof. Since $s$ is less than the injectivity radius of $V_{t}$ we may express the left side in geodesic polar coordinates and use the right hand inequality of 
Lemma 3 to get

$$
\int_{B(x, s)}\left|h-h_{B}\right|^{2} \sqrt{g(r, \xi)} d r d \sigma(\xi) \leq C_{1} \int_{B(x, s)}\left|h-h_{B}\right|^{2} r^{n-1} d r d \sigma(\xi) .
$$

Applying the regular Euclidean Poincaré inequality to the right side, the right side is bounded by

$$
C_{1} s^{2} \int_{B(x, s)}|\nabla h|^{2} r^{n-1} d r d \sigma(\xi)
$$

Applying the left hand inequality of Lemma 3 to the integral above we immediately get our lemma.

We next need a covering lemma the statement of which was motivated by the pictures in Gromov's paper [G].

Lemma 5. There exists a family of geodesic balls $\left\{B\left(x_{1}, r_{0}\right)\right\}_{i=1}^{k}$ such that

(a) $V_{t}=\bigcup_{i=1}^{k} B\left(x_{i}, r_{0}\right)$

(b) For $i \neq j, B\left(x_{i}, \frac{r_{0}}{2}\right) \cap B\left(x_{j}, \frac{r_{0}}{2}\right)$ is a set of measure zero.

(c) $k \leq C r_{0}^{-n}, C=C(f)$ independent of $t$ and $t_{0}$.

Proof. Parts (a) and (b) are trivial to accomplish. For part (c) we use a result of R. Hardt [H2], which is based on [H1]. Particularly elegant proofs have also been given by $\mathrm{H}$. Sussmann $[\mathrm{S}]$ and B. Teissier [T]. Their results state that $\mathcal{H}^{n}\left(V_{t}\right)$, the $n$-dimensional Hausdorff measure of $V_{t}$, satisfies

$$
\mathcal{H}^{n}\left(V_{t}\right) \leq C, \quad C=C(f)
$$

independent of $t$ and $t_{0}$.

Part (c) immediately follows from (b) and (2), for $V_{t} \supset \bigcup_{i-1}^{k} B\left(x_{i}, \frac{r_{0}}{2}\right)$ and thus by (b), (2) and Lemma 3,

$$
k r_{0}^{n} \leq C \mathcal{H}^{n}\left(V_{t}\right) \leq C
$$

This gives us (c).

We can now prove our theorem. 
Proof of the theorem. It will be enough to show that one can find a constant $c_{h}=c(h)$, such that for any $h \in C^{1}\left(V_{t}\right)$,

$$
\int_{V_{t}}\left|h-c_{h}\right|^{2} d \mu \leq C\left|t-t_{0}\right|^{-\alpha} \int_{V_{t}}|\nabla h|^{2} d \mu .
$$

We now denote the balls $B\left(x_{i}, r_{0}\right)$ in Lemma 5 by $B_{i}$. We simply set

$$
c_{h}=\frac{1}{\left|B_{1}\right|} \int_{B_{1}} h(r, \xi) r^{n-1} d r d \sigma(\xi) .
$$

By (a) of Lemma 5,

$$
\int_{V_{t}}\left|h-c_{h}\right|^{2} d \mu \leq \sum_{i=1}^{k} \int_{B_{i}}\left|h-c_{h}\right|^{2} d \mu .
$$

Using the notation of Lemma 4, we set

$$
h_{B_{i}}=\frac{1}{\left|B\left(x_{i}, r_{0}\right)\right|} \int_{B\left(x_{i}, r_{0}\right)} h(r, \xi) r^{n-1} d r d \sigma(\xi) .
$$

The right side of (4) is thus at most

$$
A+B=4 \sum_{i=1}^{k} \int_{B_{i}}\left|h-h_{B_{i}}\right|^{2}+4 \sum_{i=1}^{k}\left|h_{B_{i}}-c_{h}\right|^{2} \mu\left(B_{i}\right) .
$$

We apply Lemma 4 to the sum $A$ to get

$$
A \leq \sum_{i=1}^{k} 4 r_{0}^{2} \int_{B_{i}}\left|\nabla h^{2}\right| d \mu \leq 4 k_{0}^{2} \int_{V_{t}}|\nabla h|^{2} d \mu .
$$

Using (c) of Lemma 5 and inserting the estimate for $k$, we do get the right side of (3). We now estimate $B$. First note we can connect any ball $B_{i}$ to the ball $B_{1}$, by a chain of balls $B_{i, 1}=B_{1}, \ldots, B_{i, m}, \ldots, B_{i, m_{0}}=B_{i}$, where the balls $B_{i, m}$ are members of the cover in Lemma 5. Thus, keeping in mind $c_{h}=h_{B_{i, 1}}$, we have

$$
\left|h_{B_{i}}-c_{h}\right| \leq \sum_{m=1}^{m_{0}}\left|h_{B_{i, m}}-h_{B_{i, m+1}}\right| .
$$

The balls $B_{i, m}$ and $B_{i, m+1}$ are adjacent and have the same radii, $r_{0}$. Thus there exists a ball $B_{m}=B\left(x, 5 r_{0}\right)$ such that

a) $B_{m} \supset B_{i, m}$ and $B_{m} \supset B_{i, m+1}$; and

b) $\left|B_{m}\right| \leq C_{1}\left|B_{i, m}\right|$ and $\left|B_{m}\right| \leq C_{1}\left|B_{i, m+1}\right|$. 
Thus,

$$
\left|h_{B_{i, m}}-h_{B_{i, m+1}}\right| \leq\left|h_{B_{i, m}}-h_{B_{m}}\right|+\left|h_{B_{m}}-h_{B_{i, m+1}}\right| .
$$

Now, by Schwarz's inequality

$$
\left|h_{B_{i, m}}-h_{B_{m}}\right| \leq \frac{1}{\left|B_{i, m}\right|} \int_{B_{m}}\left|h-h_{B_{m}}\right| \leq\left(\frac{1}{\left|B_{i, m}\right|} \int_{B_{m}}\left|h-h_{B_{m}}\right|^{2}\right)^{1 / 2} .
$$

By (a) and (b) above $\left|B_{i, m}\right| \approx\left|B_{m}\right|$. Thus applying Lemma 3 to the last integral on the right above we may replace the last integral by

$$
\left(\frac{1}{\left|B_{m}\right|} \int_{B_{m}}\left|h-h_{B_{m}}\right|^{2} d \mu\right)^{1 / 2} \text {. }
$$

Since $5 r_{0} \leq \tau\left(V_{t}\right)$ we may apply Lemma 4 to upper bound the expression above by

$$
C_{1} r_{0}\left(\frac{1}{\left|B_{m}\right|} \int_{B_{m}}|\nabla h|^{2} d \mu\right)^{1 / 2} \text {. }
$$

Thus, we have the pair of inequalities

and

$$
\left|h_{B_{i, m}}-h_{B_{m}}\right| \leq C_{1} r_{0}\left(\frac{1}{\left|B_{m}\right|} \int_{B_{m}}|\nabla h|^{2} d \mu\right)^{1 / 2}
$$

$$
\left|h_{B_{i, m+1}}-h_{B_{m}}\right| \leq C_{1} r_{0}\left(\frac{1}{\left|B_{m}\right|} \int_{B_{m}}|\nabla h|^{2} d \mu\right)^{1 / 2} .
$$

From (7) and the pair of inequalities above we get

$$
\left|h_{B_{i, m+1}}-h_{B_{i, m}}\right| \leq C_{1} r_{0}\left|B_{m}\right|^{-1 / 2}\left(\int_{B_{m}}|\nabla h|^{2} d \mu\right)^{1 / 2} .
$$

Inserting this in (6), and remembering that $\left|B_{m}\right| \approx C_{1} r_{o}^{n}$ and $m_{0} \leq k$, we get

$$
\left|h_{B_{i}}-c_{h}\right| \leq C r_{0}^{-(n-2) / 2} k\left(\int_{V_{t}}|\nabla h|^{2} d \mu\right)^{1 / 2} .
$$

We now insert this into the expression $B$ in (5), and recalling from Lemma 3 that $\mu\left(B_{i}\right) \approx C_{1} r_{0}^{n}$, we get

$$
B \leq C_{1} r_{0}^{2} k^{3} \int_{V_{t}}|\nabla h|^{2} d \mu .
$$

Thus summing up $A$ and $B$,

$$
\int_{V_{t}}\left|h-c_{h}\right|^{2} d \mu \leq C_{1} r_{0}^{2} k^{3} \int_{V_{t}}|\nabla h|^{2} d \mu .
$$

Since $k \leq C r_{0}^{-n}$ and $r_{0}=\frac{C\left|t-t_{0}\right|^{-\beta}}{100}$, we easily have (3) with $\alpha=\alpha_{2}(3 n-2)$, $\alpha_{2}=2 \alpha_{1}$. This proves the theorem. 


\section{Acknowledgement}

I am deeply indebted to Paulo Cordaro for bringing this problem to my attention. I also wish to thank F. Treves for his encouragement and interest in this work.

\section{References}

[C] I. Chavel, Eigenvalues in Riemannian Geometry, Academic Press, 1984.

$[\mathrm{CH}]$ P. Cordaro and J. Hounie, On local solvability of underdetermined systems of vector fields, Amer. J. of Math. 112 (1990), 243-270.

[G] M. Gromov, Spectral Geometry of semi-algebraic sets, Ann. Inst. Fourier (Grenoble) 42 (1992), 249-274.

[H1] R. Hardt, Slicing and intersection theory for chains associated with real analytic varieties, Acta Math. 129 (1972), 75-136.

[H2] R. Hardt, Some analytic bounds for subanalytic sets, Differential Geometry and Control Theory (R.W. Brockett, R.S. Millman and H.J. Sussmann, eds.), Birkhauser, 1983, pp. 259-267.

[S] H. Sussmann, Real analytic desingularization and subanalytic sets: an elementary approach, Trans. Amer. Math. Soc. 317 (1990), 417-461.

[T] B. Teissier, Sur trois questions de finitude en geómetric analytique reele, appendix to the paper of F. Treves, Acta Math. 151 (1983), 39-48.

[Tr] F. Treves, On the local solvability and the local integrability of systems of vector fields, Acta Math. 151 (1983), 1-38.

Dept. of Mathematics, Rutgers University, New Brunswick, NJ 08903.

E-mail address: chanillo@math.rutgers.edu 